\title{
Regularity and approximation of systems arising in electromagnetic interrogation of dielectric materials
}

\author{
H.T. Banks ${ }^{1}$ and Jun Zou ${ }^{2}$
}

\begin{abstract}
In this paper we are concerned with a mathematical model which describes the electromagnetic interrogation of dielectric materials. We address the well-posedness of the system and regularity of solutions. Then we propose a semi-discrete finite element scheme for approximating the system and prove weak convergence under very mild regularity assumptions on the solutions of the original system. We also establish that the solution has higher regularity in time even though the input source may be a windowed signal of distributional type. This regularity is then utilized to show the strong convergence of the finite element solution.
\end{abstract}

\section{Introduction}

We first formulate a mathematical model describing the electromagnetic interrogation of dielectric materials. Let $\mathbf{E}$ and $\mathbf{H}$ be the intensities of electric and magnetic field, $\mathbf{D}$ and $\mathbf{B}$ be the electric and magnetic flux densities, $\mathbf{J}$ the current density. The electric and magnetic polarization are denoted as

\footnotetext{
${ }^{1}$ Center for Research in Scientific Computation, North Carolina State University, Raleigh, NC 27695. E-mail: htbanks@eos.ncsu.edu. The work of this author was partially supported by the Air Force Office of Scientific Research under grants AFOSR F49620-951-0236 and AFOSR F49620-98-1-0180.

${ }^{2}$ Department of Mathematics, The Chinese University of Hong Kong, Shatin, N.T., Hong Kong. E-mail: zou@math.cuhk.edu.hk. The work of this author was partially supported by Hong Kong RGC Grant No. CUHK 4004/98P.
} 
$\mathbf{P}$ and $\mathbf{M}$. Then the mathematical model to be discussed in this paper is formulated as follows:

$$
\begin{aligned}
-\frac{\partial \mathbf{D}}{\partial t}+\nabla \times \mathbf{H} & =\mathbf{J}, \\
\frac{\partial \mathbf{B}}{\partial t}+\nabla \times \mathbf{E} & =0, \\
\nabla \cdot \mathbf{D} & =\rho_{e m}, \\
\nabla \cdot \mathbf{B} & =0,
\end{aligned}
$$

along with the basic constitutive relation (Ohm's law) for a conductive dielectric material and the the electric and magnetic polarizations:

$$
\begin{aligned}
\mathbf{J} & =\sigma \mathbf{E}, \\
\mathbf{D} & =\varepsilon \mathbf{E}+\mathbf{P}, \\
\mathbf{B} & =\mu_{0} \mathbf{H}+\mathbf{M} .
\end{aligned}
$$

Among the media of primary interest to us are biological ones; hence we choose to ignore the magnetic effect, i.e., we assume $\mathbf{M}=0$. We will adopt the following general polarization model in terms of a convolution of the electric field with an impulse response function $g$ (sometimes called a displacement susceptibility kernel):

$$
\mathbf{P}(t, z)=\int_{0}^{t} g(t-s, z) \mathbf{E}(s, z) d s
$$

whose first and second order derivatives with respect to $t$ are given by

$$
\begin{aligned}
& \dot{\mathbf{P}}(t)=g(0) \mathbf{E}(t)+\int_{0}^{t} \dot{g}(t-s) \mathbf{E}(s) d s \\
& \ddot{\mathbf{P}}(t)=g(0) \dot{E}(t)+\dot{g}(0) \mathbf{E}(t)+\int_{0}^{t} \ddot{g}(t-s) \mathbf{E}(s) d s .
\end{aligned}
$$

The above polarization model has been used in the literature on electromagnetic wave propagation, e.g. in $[1,7,12]$. It includes as special cases systems with memory (or time delay) and systems with hysteresis as well as the usual Debye, Lorentz and higher order differential equation polarization laws (see $[3,4,8]$ ). We note that for biological media (among others), one should probably replace Ohm's law (1.5) with a constitutive law similar to our polarization law (1.8), e.g., see [1] or [9] (pp. 14-16). While the resulting model 
would still be amenable to our treatment here, inclusion of a conductivity susceptibility kernel would add to the technical notation. We have chosen for the sake of exposition to restrict our formulation to the Ohm's law version as given in (1.5).

We assume that there is a special interrogating input signal, namely a planar electromagnetic wave normaly incident on a slab of material contained in the interval $\Omega=\left[\begin{array}{ll}\tilde{z}_{0}, & \tilde{z}_{1}\end{array}\right]$ with faces parallel to the $x y$-plane, see Fig. 1. The electric field is polarized with oscillations in the $x z$-plane only, i.e., $\mathbf{E}=$ $E(t, z) \mathbf{i}$, where $\mathbf{i}$ is the unit direction in the $x$-axis.

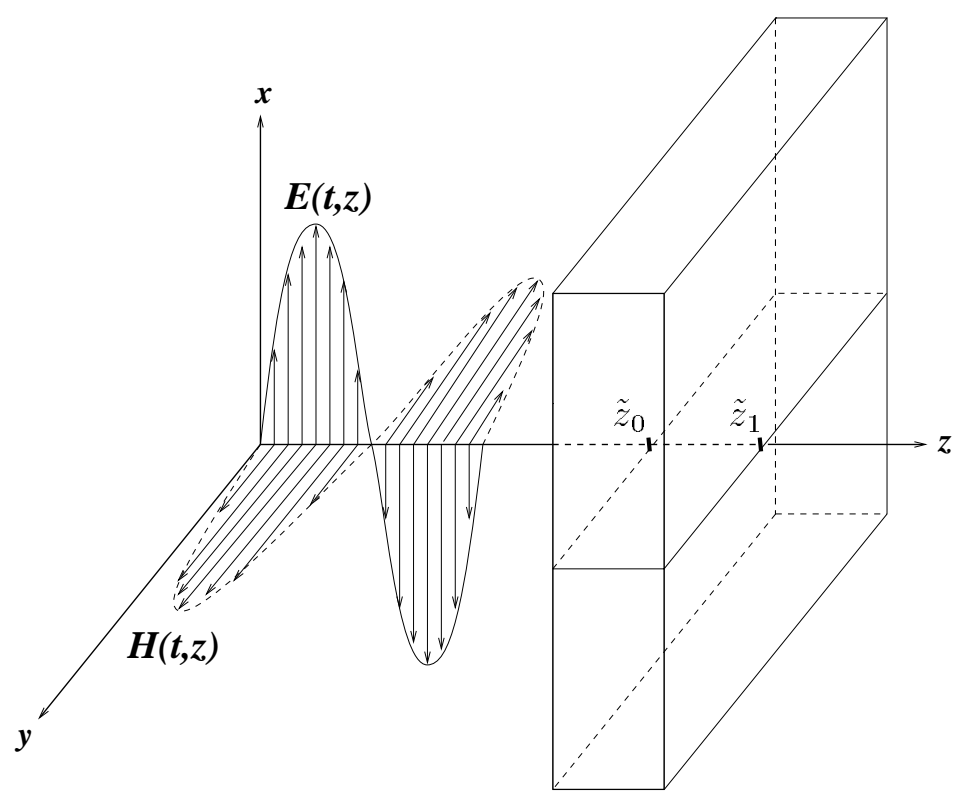

Figure 1: Geometry of the physical problem

Under these assumptions, we know that the electric field $\mathbf{E}(t, \mathbf{x})$ is parallel to the $x$-axis at all points in the region outside the slab domain $\Omega$, i.e., $\Omega_{0}=\left(0, \tilde{z}_{0}\right)$, which we assume is just air, and that the magnetic field $\mathbf{H}(t, \mathbf{x})$ is always parallel to the $y$-axis, so we have

$$
\mathbf{E}(t, \mathbf{x})=(E(t, z), 0,0)^{\top}, \quad \mathbf{H}(t, \mathbf{x})=(0, H(t, z), 0)^{\top} .
$$

Moreover, the electric flux density $\mathbf{D}$ and polarization $\mathbf{P}$ will inherit this uniform directional property from $\mathbf{E}$ and hence are denoted hereafter by 
their scalar magnitudes $D$ and $P$ in the $x$ direction. Using these notations, the Maxwell's equations (1.1)-(1.3) reduces to the system

$$
\begin{aligned}
\mu_{0} \frac{\partial H}{\partial t}+\frac{\partial E}{\partial z} & =0 \\
\frac{\partial D}{\partial t}-\frac{\partial H}{\partial z} & =\sigma E+J_{s}
\end{aligned}
$$

Eliminating the magnetic field $H$ from the two equations, we obtain the electric field equation

$$
\mu_{0} \ddot{D}+\mu_{0} \sigma \dot{E}-E^{\prime \prime}=-\mu_{0} \dot{J}_{s} .
$$

We remark that throughout we adopt the standard notation

$$
\dot{E}=\frac{\partial E}{\partial t}, \quad \ddot{E}=\frac{\partial^{2} E}{\partial t^{2}}, \quad E^{\prime}=\frac{\partial E}{\partial z}, \quad E^{\prime \prime}=\frac{\partial^{2} E}{\partial z^{2}}, \quad \text { etc. }
$$

By using (1.6) with $\varepsilon=\varepsilon_{0}\left(1+\left(\varepsilon_{r}-1\right) \chi_{\Omega}\right)$ (this accounts for instantaneous effects of polarization, see [3]), and (1.10), we can readily derive the equation which will be investigated in this paper:

$$
\tilde{\varepsilon}_{r}(z) \ddot{E}+\gamma(z) \ddot{E}+\beta(z) E+G(E)-c^{2} E^{\prime \prime}=J
$$

where $c^{2}=1 /\left(\varepsilon_{0} \mu_{0}\right), \tilde{\varepsilon}_{r}(z)=1+\left(\varepsilon_{r}-1\right) \chi_{\Omega}(z)$, and

$$
\begin{aligned}
\alpha(t, z) & =\frac{1}{\varepsilon_{0}} \ddot{g}(t, z) \chi_{\Omega}(z), \quad \beta(z)=\frac{1}{\varepsilon_{0}} \dot{g}(0, z) \chi_{\Omega}(z), \\
\gamma(z) & =\frac{1}{\varepsilon_{0}}(\sigma(z)+g(0, z)) \chi_{\Omega}(z), \quad J(t, z)=-\frac{1}{\varepsilon_{0}} \dot{J}_{s}, \\
G(E)(t, z) & =\int_{0}^{t} \alpha(t-s, z) E(s, z) d s .
\end{aligned}
$$

Here $\chi_{\Omega}(z)$ is the characteristic function of $\Omega=\left[\tilde{z}_{0}, \tilde{z}_{1}\right]$, and $\varepsilon_{r} \geq 1$ is the relative dielectric permittivity. Note that $\tilde{\varepsilon}_{r}(z)=1$ for $z \in\left[0, \tilde{z}_{0}\right)$ and $\tilde{\varepsilon}_{r}(z)=$ $\varepsilon_{r} \geq 1$ for $z \in\left[\tilde{z}_{0}, \tilde{z}_{1}\right]$ and all the coefficient functions $\alpha, \beta$ and $\gamma$ are only piecewise smooth, having jumps across the interface $z=\tilde{z}_{0}$. The source current density function is under our control, and we assume it takes the form

$$
J_{s}(t, z)=\delta(z) \mu(t)
$$


with $\mu(t)$ being a smooth function of $t$ and

$$
\mu(0)=\dot{\mu}(0)=0 .
$$

These assumptions are valid if we have a point source input signal (an antenna) at the location $z=0$ for which the power is switched on after a small time period of delay.

Regarding the boundary conditions, we assume the following physical situation. At the boundary $z=0$ where an input signal originates, we place an absorbing boundary condition to prevent the reflection of waves. Mathematically this can be expressed as

$$
\dot{E}(t, 0)=c E^{\prime}(t, 0) .
$$

We assume the location of the front boundary $\tilde{z}_{0}$ of the slab material is known while the back boundary $\tilde{z}_{1}$ is unknown and presumed to be backed by a supraconductive material with perfect conductivity, thus the back boundary conditions are given by $([2], \mathrm{p} .26)$

$$
\mathbf{E} \times \mathbf{n}=0, \quad \mathbf{H} \times \mathbf{n}=0, \quad \mathbf{D} \cdot \mathbf{n}=0, \quad \mathbf{B} \cdot \mathbf{n}=0
$$

where $\mathbf{n}$ is the outward normal to the slab. For our fields $\mathbf{E}, \mathbf{H}$ and the constitutive laws (1.6), (1.7) and (1.8), the conditions $\mathbf{D} \cdot \mathbf{n}=0, \mathbf{B} \cdot \mathbf{n}=0$ are automatically satisfied while the other conditions reduce to

$$
E\left(t, \tilde{z}_{1}\right)=H\left(t, \tilde{z}_{1}\right)=0 .
$$

Since we have eliminated $H$ from the system (1.11), the only relevant boundary condition for the supraconductive back boundary is $E\left(t, \tilde{z}_{1}\right)=0$.

In many applications, the coefficient functions $\alpha, \beta$ and $\gamma$ in (1.11) and the back boundary of the slab domain at $z=\tilde{z}_{1}$ are unknown. They can be identified numerically from data and this identification problem has been investigated in $[3,4,5]$ successfully using finite element schemes similar to that proposed below in Section 3. In this paper we assume that the coefficient functions $\alpha, \beta$ and $\gamma$ and the back boundary $z=\tilde{z}_{1}$ are known and we are interested in the well-posedness and regularity of the forward problem (1.11) and the convergence analysis of its finite element discretization.

To avoid notational difficulties, we use a piecewise linear transformation to map the slab domain $\left(\tilde{z}_{0}, \tilde{z}_{1}\right)$ into $\left(\tilde{z}_{0}, 1\right)$ while keeping the external domain $\left(0, \tilde{z}_{0}\right)$ invariant. Since the investigation of the transformed system is 
principally the same as the study for the original system, we will assume the original system is defined on the transformed domain [0,1]. Then summarizing the above derivations, we can formulate the aformentioned model problem as the following system on the time-space domain $Q_{T}=(0, T) \times(0,1)$ :

$$
\begin{aligned}
& \tilde{\varepsilon}_{r}(z) \ddot{E}+\gamma(z) \ddot{E}+\beta(z) E+G(E)-c^{2} E^{\prime \prime}=J(t, z) \text { on } Q_{T}, \\
& \dot{E}(t, 0)=c E^{\prime}(t, 0) \text { and } E(t, 1)=0 \quad \text { on } \quad(0, T)
\end{aligned}
$$

to which are added the initial conditions

$$
E(0, z)=\Phi(z) \quad \text { and } \quad \dot{E}(0, z)=\Psi(z) \quad \text { on } \quad(0,1)
$$

Throughout the paper, we will make the following assumptions:

$$
\beta \in L^{\infty}(0,1), \quad \gamma \in L^{\infty}(0,1) \quad \text { and } \quad \alpha \in H^{1}\left(0, T ; L^{\infty}(0,1)\right) .
$$

\section{Well-posedness and regularity}

In this section we address the well-posedness and regularity for the initialboundary value problem (1.16)-(1.18). Due to the piecewise smoothness of the coefficient functions and the input signal of distributional type, we can not expect classical solutions for the problem. Instead we will consider its weak solutions. For this, we introduce the following two Hilbert spaces

$$
H=L^{2}(0,1), \quad V=H_{R}^{1}(0,1)=\left\{v \in H^{1}(0,1) ; v(1)=0\right\} .
$$

We will use $V^{*}$ to denote the dual space of $V$ and $\langle\cdot, \cdot\rangle$ to denote their duality pairing, which is assumed to be a continuous extension of the scalar product $(\cdot, \cdot)$ in $H=L^{2}(0,1)$. The standard norms in $H$ and $V$ will be written as $\|\cdot\|$ and $\|\cdot\|_{V}$. We will also have occasions to use the Sobolev space $H_{R}^{m}(0,1)=H_{R}^{1}(0,1) \cap H^{m}(0,1)$ for some positive integer $m$.

We next present the weak formulation of the problem (1.16)-(1.18). Multiplying equation (1.16) by a test function $\phi \in V$ and then using integration by parts we immediately derive the weak form:

Find $E \in H^{2}\left(0, T ; V^{*}\right) \cap H^{1}(0, T ; H) \cap L^{2}(0, T ; V)$ such that

$$
E(0, z)=\Phi(z), \quad \dot{E}(0, z)=\Psi(z), \quad z \in(0,1)
$$


and for a.e. $t \in(0, T)$, the following holds

$$
\begin{aligned}
& \left\langle\hat{\varepsilon}_{r} \ddot{E}(t), \phi\right\rangle+(\gamma \dot{E}(t), \phi)+(\tilde{\beta} E(t), \phi)+(G(E)(t), \phi)+c^{2}\left(E^{\prime}(t), \phi^{\prime}\right) \\
& +c \dot{E}(t, 0) \phi(0)=\langle J(t), \phi\rangle+\left(k_{0} E(t), \phi\right) \quad \forall \phi \in V .
\end{aligned}
$$

We remark that we have added the term $\left(k_{0} E(t), \phi\right)$ in both sides of $(2.2)$ for later convenience. Here $k_{0}$ is a positive constant chosen such that

$$
\tilde{\beta}(z)=\beta(z)+k_{0} \geq \frac{k_{0}}{2}, \quad \text { for all } z \in[0,1] .
$$

Regarding the existence of a unique solution for the problem (2.1)-(2.2), we have (see Banks-Buksas [3])

Theorem 2.1 Suppose that $J \in H^{1}\left(0, T ; V^{*}\right), \beta, \gamma \in L^{\infty}(0,1)$ and $\alpha \in$ $H^{1}\left(0, T ; L^{\infty}(0,1)\right)$ with $\alpha, \beta$ and $\gamma$ vanishing outside $\left[\tilde{z}_{0}, 1\right]$. Then for $\Phi \in$ $V=H_{R}^{1}(0,1)$ and $\Psi \in H=L^{2}(0,1)$ there exists a unique solution $E$ to the variational problem (2.1)-(2.2) with $E(\cdot, 0) \in H^{1}(0, T)$.

We note that we can derive the following a priori estimates for any possible solutions $E$ of problem (2.1)-(2.2):

$$
\|\dot{E}\|_{L^{2}(0, T ; H)}+\|E\|_{L^{2}(0, T ; V)}+\|\dot{E}(\cdot, 0)\|_{L^{2}(0, T)} \leq C(\Phi, \Psi, J)
$$

with constant $C(\Phi, \Psi, J)$ given by

$$
C(\Phi, \Psi, J)=C_{0}\left\{\|\Psi\|+\|\Phi\|_{V}+\|J\|_{H^{1}\left(0, T ; V^{*}\right)}\right\}
$$

and $C_{0}$ is a constant dependent only on the coefficient functions $\alpha, \beta$ and $\gamma$. The arguments for the above a priori bounds (which are given in [3]) are very similar to those for obtaining the bound (2.25) in Theorem 2.2 below, so we will not give details here.

Now note the uniqueness of the solutions follows immediately from the a priori estimate (2.3)-(2.4) by considering the error equation of the difference of two possible solutions $E_{1}$ and $E_{2}$ to (2.1)-(2.2) using the fact that $C(\Phi, \Psi, J)=0$ in this case. The existence of the solutions, which was established in [3], is also a by-product of the weak convergence of the finite element solutions in Theorem 4.1 below (i.e., the limiting function is the solution of (2.1)-(2.2)) 
For achieving some type of strong convergence of the finite element approximation to be introduced later, we require additional regularity of the solution $E$ in terms of time $t$. This is possible to obtain since the source and coefficient functions are smooth in terms of $t$, though it is impossible to have more regularity than $H^{1}(0,1)$ in space due to the source input of distributional type and the piecewise smoothness of the coefficients in terms of $z$.

We are going to establish the following result.

Theorem 2.2 Suppose that $J(t, z)=\mu(t) \delta(z)$ with $\mu \in H^{2}(0, T)$ and $\mu(0)=$ $\dot{\mu}(0)=0, \beta, \gamma \in L^{\infty}(0,1)$ and $\alpha \in H^{2}\left(0, T ; L^{\infty}(0,1)\right)$ with $\alpha, \beta$ and $\gamma$ vanishing outside $\left[\tilde{z}_{0}, 1\right]$. Moreover we assume that $\Phi \in H_{R}^{3}(0,1), \Psi \in H_{R}^{2}(0,1)$ and they satisfy the consistency conditions $\Psi(0)=c \Phi^{\prime}(0)$ and $\Psi^{\prime}(0)=c \Phi^{\prime \prime}(0)$. Then the unique solution $E$ of the variational problem (2.1)-(2.2) has the following regularity

$$
E \in H^{3}\left(0, T ; V^{*}\right) \cap H^{2}(0, T ; H) \cap H^{1}(0, T ; V) .
$$

Proof. Our approach is to first smooth all the given data and then solve the smoothed problem to obtain solutions with some added regularity. We then pass to the limit by letting the smoothing parameter tend to zero and achieve the desired regularity. Following notational tradition in analysis, we shall use $\varepsilon$ as our smoothing parameter in this section while warning readers that it is not related to the dielectric permittivity parameter $\varepsilon$ (again traditional notation for the electromagnetic literature) of Section 1.

Let $0<\varepsilon<\tilde{z}_{0}$ be given. Since $\mu \in H^{2}(0, T), \Phi \in H_{R}^{3}(0,1)$ and $\Psi \in$ $H_{R}^{2}(0,1)$, we can construct $\mu_{\varepsilon} \in H^{3}(0, T), \Phi_{\varepsilon} \in H_{R}^{3}(0,1)$ and $\Psi_{\varepsilon} \in H_{R}^{2}(0,1)$ such that

$$
\begin{array}{lll}
\mu_{\varepsilon}(0)=\mu(0), & \Psi_{\varepsilon}(0)=\Psi(0), & \Phi_{\varepsilon}^{\prime}(0)=\Phi^{\prime}(0) \\
\dot{\mu_{\varepsilon}}(0)=\dot{\mu}(0), & \Psi_{\varepsilon}^{\prime}(0)=\Psi^{\prime}(0), & \Phi_{\varepsilon}^{\prime \prime}(0)=\Phi^{\prime \prime}(0)
\end{array}
$$

and

$$
\left\|\mu_{\varepsilon}-\mu\right\|_{H^{2}(0, T)} \leq \varepsilon, \quad\left\|\Phi_{\varepsilon}-\Phi\right\|_{H^{2}(0,1)} \leq \varepsilon, \quad\left\|\Psi_{\varepsilon}-\Psi\right\|_{H^{1}(0,1)} \leq \varepsilon .
$$

Define an approximating source function

$$
J_{\varepsilon}(t, z)=\frac{2(\varepsilon-z)}{\varepsilon^{2}} \mu_{\varepsilon}(t) \text { for } z \in[0, \varepsilon] ; \quad J_{\varepsilon}(t, z)=0 \text { for } z \in[\varepsilon, 1] .
$$


It is easy to show that

$$
\left\|J-J_{\varepsilon}\right\|_{H^{2}\left(0, T ; V^{*}\right)} \rightarrow 0 \quad \text { as } \quad \varepsilon \rightarrow 0 .
$$

Now consider the following smoothed problem:

Find $E_{\varepsilon} \in H^{2}\left(0, T ; V^{*}\right) \cap H^{1}(0, T ; H) \cap L^{2}(0, T ; V)$ such that

$$
E_{\varepsilon}(0, z)=\Phi_{\varepsilon}(z), \quad \dot{E}_{\varepsilon}(0, z)=\Psi_{\varepsilon}(z), \quad z \in(0,1)
$$

and for a.e. $t \in(0, T)$, the following holds

$$
\begin{aligned}
& \left\langle\tilde{\varepsilon}_{r} \ddot{E}_{\varepsilon}(t), \phi\right\rangle+\left(\gamma \dot{E}_{\varepsilon}(t), \phi\right)+\left(\tilde{\beta} E_{\varepsilon}(t), \phi\right)+\left(G\left(E_{\varepsilon}\right)(t), \phi\right)+c^{2}\left(E_{\varepsilon}^{\prime}(t), \phi^{\prime}\right) \\
& +c \dot{E}_{\varepsilon}(t, 0) \phi(0)=\left\langle J_{\varepsilon}(t), \phi\right\rangle+\left(k_{0} E_{\varepsilon}(t), \phi\right) \forall \phi \in V .
\end{aligned}
$$

The same argument as for Theorem 2.1 leads to the existence of a unique solution $E_{\varepsilon}$ to the problem (2.11)-(2.12). We next show this solution has the following higher regularity:

$$
E_{\varepsilon} \in H^{3}\left(0, T ; V^{*}\right) \cap H^{2}(0, T ; H) \cap H^{1}(0, T ; V) .
$$

To do so, we formally differentiate both sides of equation (2.12) with respect to $t$ and introduce a new variable $v_{\varepsilon}$ for $\dot{E}_{\varepsilon}$ to obtain

$$
\begin{aligned}
& \left\langle\tilde{\varepsilon}_{r} \ddot{v}_{\varepsilon}(t), \phi\right\rangle+\left(\gamma \dot{v}_{\varepsilon}(t), \phi\right)+\left(\tilde{\beta} v_{\varepsilon}(t), \phi\right)+c^{2}\left(v_{\varepsilon}^{\prime}(t), \phi^{\prime}\right) \\
& +c \dot{v}_{\varepsilon}(t, 0) \phi(0)+\left(\alpha(0) \int_{0}^{t} v_{\varepsilon}(s) d s+\int_{0}^{t} \dot{\alpha}(t-s) \int_{0}^{s} v_{\varepsilon}\left(s^{\prime}\right) d s^{\prime} d s, \phi\right) \\
& =\left\langle\dot{J}_{\varepsilon}(t), \phi\right\rangle+\left(k_{0} v_{\varepsilon}(t), \phi\right)-\left((2 \alpha(0)-\alpha(t)) \Phi_{\varepsilon}, \phi\right) \forall \phi \in V .
\end{aligned}
$$

We supplement the equation (2.14) with the following initial conditions

$$
v_{\varepsilon}(0, z)=\Psi_{\varepsilon}(z), \quad \dot{v}_{\varepsilon}(0, z)=Z_{\varepsilon}(z)
$$

with $Z_{\varepsilon}=-\tilde{\varepsilon}_{r}^{-1}\left(\gamma \Psi_{\varepsilon}+\beta \Phi_{\varepsilon}-c^{2} \Phi_{\varepsilon}^{\prime \prime}\right)$ (note that $\left.J_{\varepsilon}(0)=0\right)$. It is easy to verify that $\Psi_{\varepsilon} \in V$ and $Z_{\varepsilon} \in H$, so we can apply the same argument as for Theorem 2.1 to establish the existence of a unique solution $v_{\varepsilon}$ to the problem (2.14)-(2.15) and

$$
v_{\varepsilon} \in H^{2}\left(0, T ; V^{*}\right) \cap H^{1}(0, T ; H) \cap L^{2}(0, T ; V) .
$$


We next verify that $v_{\varepsilon}=\dot{E}_{\varepsilon}$. For this purpose, we define

$$
w_{\varepsilon}(t)=\Phi_{\varepsilon}+\int_{0}^{t} v_{\varepsilon}(s) d s .
$$

Clearly we see

$$
\begin{aligned}
& \dot{w}_{\varepsilon}(t)=v_{\varepsilon}(t), \quad \ddot{w}_{\varepsilon}(t)=\dot{v}_{\varepsilon}(t), \\
& w_{\varepsilon}(0)=\Phi_{\varepsilon}, \quad \dot{w}_{\varepsilon}(0)=\Psi_{\varepsilon} .
\end{aligned}
$$

By integrating both sides of $(2.14)$ over $(0, t)$ and using $(2.15)$, we obtain

$$
\begin{aligned}
\left\langle\tilde{\varepsilon}_{r} \ddot{w}_{\varepsilon}(t), \phi\right\rangle+ & \left(\gamma \dot{w}_{\varepsilon}(t), \phi\right)+\left(\tilde{\beta} w_{\varepsilon}(t), \phi\right)+c^{2}\left(w_{\varepsilon}^{\prime}(t), \phi^{\prime}\right) \\
& +c \dot{w}_{\varepsilon}(t, 0) \phi(0)+\left(\int_{0}^{t} \alpha(t-s) w_{\varepsilon}(s) d s, \phi\right) \\
=\left\langle J_{\varepsilon}(t), \phi\right\rangle & +\left\{\left(\tilde{\varepsilon}_{r} Z_{\varepsilon}, \phi\right)+\left(\gamma \Psi_{\varepsilon}, \phi\right)+\left(\beta \Phi_{\varepsilon}, \phi\right)\right. \\
& \left.+c^{2}\left(\Phi_{\varepsilon}^{\prime}, \phi^{\prime}\right)+c \Psi_{\varepsilon}(0) \phi(0)-\left(J_{\varepsilon}(0), \phi\right)\right\}, \quad \forall \phi \in V \\
\equiv:(\mathrm{I})_{1}+(\mathrm{I})_{2} &
\end{aligned}
$$

Using (2.15), integration by parts and the consistency condition, we can readily show that $(\mathrm{I})_{2}=0$. Then comparing equations (2.18)-(2.19) with equations (2.11)-(2.12) and using the uniqueness of solutions to equations (2.11)-(2.12) we have

$$
w_{\varepsilon}(t)=E_{\varepsilon}(t) \quad \text { or } \quad v_{\varepsilon}(t)=\dot{E}_{\varepsilon}(t)
$$

And hence (2.13) follows from the regularity of $v_{\varepsilon}$ in (2.16).

Again we formally differentiate both sides of equation (2.14) with respect to $t$ and introduce a variable $u_{\varepsilon}$ for $\dot{v}_{\varepsilon}$ to obtain

$$
\begin{aligned}
& \left\langle\tilde{\varepsilon}_{r} \ddot{u}_{\varepsilon}(t), \phi\right\rangle+\left(\gamma \dot{u}_{\varepsilon}(t), \phi\right)+\left(\tilde{\beta} u_{\varepsilon}(t), \phi\right)+c^{2}\left(u_{\varepsilon}^{\prime}(t), \phi^{\prime}\right)+c \dot{u}_{\varepsilon}(t, 0) \phi(0) \\
& +\left(G_{1}\left(u_{\varepsilon}\right)(t), \phi\right)=\left\langle\ddot{J}_{\varepsilon}(t), \phi\right\rangle+\left(k_{0} u_{\varepsilon}(t), \phi\right)+\left(\tilde{J}_{\varepsilon}(t), \phi\right) \quad \forall \phi \in V
\end{aligned}
$$

with

$G_{1}\left(u_{\varepsilon}\right)(t)=\int_{0}^{t}\left\{\alpha(0) u_{\varepsilon}(s)+\dot{\alpha}(0) \int_{0}^{s} u_{\varepsilon}(\tau) d \tau+\ddot{\alpha}(t-s) \int_{0}^{s} \int_{0}^{s^{\prime}} u_{\varepsilon}(\tau) d \tau d s^{\prime}\right\} d s$ 
and

$$
\tilde{J}_{\varepsilon}(t)=\dot{\alpha}(t) \Phi_{\varepsilon}-\alpha(0) \Psi_{\varepsilon}-t \dot{\alpha}(0) \Psi_{\varepsilon}-\Psi_{\varepsilon} \int_{0}^{t} s \ddot{\alpha}(t-s) d s .
$$

By supplementing equation (2.20) with the initial conditions

$$
u_{\varepsilon}(0)=Z_{\varepsilon}, \quad \dot{u}_{\varepsilon}(0)=Y_{\varepsilon}
$$

where $Y_{\varepsilon}=-\tilde{\varepsilon}_{r}^{-1}\left(\alpha(0) \Phi_{\varepsilon}+\beta \Psi_{\varepsilon}+\gamma Z_{\varepsilon}-c^{2} \Psi_{\varepsilon}^{\prime \prime}\right)$, we can argue in a same way as we did above that the solution $u_{\varepsilon}$ of equation $(2.20)$ really equals $\dot{v}_{\varepsilon}$, and

$$
\ddot{E}_{\varepsilon}=\dot{v}_{\varepsilon} \in H^{2}\left(0, T ; V^{*}\right) \cap H^{1}(0, T ; H) \cap L^{2}(0, T ; V) .
$$

Here we need to use the facts that $\gamma(0)=\sigma(0)=\beta(0)=0, \tilde{\varepsilon}_{r}(0)=1$ (by definition) and the consistency condition $\Psi^{\prime}(0)=c \Phi^{\prime \prime}(0)$.

Now we are in a position to give some bounds on $E_{\varepsilon}$ which are independent of $\varepsilon$. Recall $v_{\varepsilon}=\dot{E}_{\varepsilon}$. Integrating both sides of $(2.14)$ over $(0, t)$ and then taking $\phi=\ddot{E}_{\varepsilon}$ (this is possible by the regularity (2.21)) we obtain

$$
\begin{aligned}
& \frac{1}{2}\left\{\left(\tilde{\varepsilon}_{r} \ddot{E}_{\varepsilon}(t), \ddot{E}_{\varepsilon}(t)\right)+\left(\tilde{\beta} \dot{E}_{\varepsilon}(t), \dot{E}_{\varepsilon}(t)\right)+c^{2}\left\|\left(\dot{E}_{\varepsilon}(t)\right)^{\prime}\right\|^{2}\right\}+c \int_{0}^{t}\left|\ddot{E}_{\varepsilon}(\tau, 0)\right|^{2} d \tau \\
= & d_{\varepsilon}+\int_{0}^{t}\left(\dot{J}_{\varepsilon}(\tau), \ddot{E}_{\varepsilon}(\tau)\right) d \tau-\int_{0}^{t}\left(\gamma \ddot{E}_{\varepsilon}(\tau), \ddot{E}_{\varepsilon}(\tau)\right) d \tau+k_{0} \int_{0}^{t}\left(\dot{E}_{\varepsilon}(\tau), \ddot{E}_{\varepsilon}(\tau)\right) d \tau \\
& +\int_{0}^{t}\left((2 \alpha(0)-\alpha(\tau)) \Phi_{\varepsilon}, \ddot{E}_{\varepsilon}(\tau)\right) d \tau-\int_{0}^{t}\left(G_{2}\left(\dot{E}_{\varepsilon}\right)(\tau), \ddot{E}_{\varepsilon}(\tau)\right) d \tau
\end{aligned}
$$

with

$$
\begin{aligned}
& d_{\varepsilon}=\frac{1}{2}\left\{\left(\tilde{\varepsilon}_{r} Z_{\varepsilon}, Z_{\varepsilon}\right)+\left(\tilde{\beta} \Psi_{\varepsilon}, \Psi_{\varepsilon}\right)+c^{2}\left\|\Psi_{\varepsilon}^{\prime}\right\|^{2}\right\} \\
& G_{2}\left(v_{\varepsilon}\right)(t)=\alpha(0) \int_{0}^{t} v_{\varepsilon}(s) d s+\int_{0}^{t} \dot{\alpha}(t-s) \int_{0}^{s} v_{\varepsilon}\left(s^{\prime}\right) d s^{\prime} d s .
\end{aligned}
$$

With integration by parts the second term in the right-hand side of $(2.22)$ can be written as

$$
\int_{0}^{t}\left(\dot{J}_{\varepsilon}(\tau), \ddot{E}_{\varepsilon}(\tau)\right) d \tau=-\int_{0}^{t}\left(\ddot{J}_{\varepsilon}(\tau), \dot{E}_{\varepsilon}(\tau)\right) d \tau+\left(\dot{J}_{\varepsilon}(t), \dot{E}_{\varepsilon}(t)\right)-\left(\dot{J}_{\varepsilon}(0), \dot{E}_{\varepsilon}(0)\right) .
$$


This with (2.22) and the Cauchy-Schwarz inequality yields immediately

$$
\begin{aligned}
& \left\|\ddot{E}_{\varepsilon}(t)\right\|^{2}+\beta_{c}\left\|\dot{E}_{\varepsilon}(t)\right\|_{V}^{2}+2 c \int_{0}^{t}\left|\ddot{E}_{\varepsilon}(\tau, 0)\right|^{2} d \tau \\
\leq & 2 d_{\varepsilon}+\frac{\beta_{c}}{2}\left\|\dot{E}_{\varepsilon}(t)\right\|_{V}^{2}+3 \bar{\alpha} t\left\|\Phi_{\varepsilon}\right\|^{2}+C\left\|J_{\varepsilon}\right\|_{H^{2}\left(0, T ; V^{*}\right)}^{2} \\
& +C \int_{0}^{t}\left(\left\|\dot{E}_{\varepsilon}(\tau)\right\|^{2}+\left\|\ddot{E}_{\varepsilon}(\tau)\right\|^{2}\right) d \tau
\end{aligned}
$$

where we have used the fact $\dot{J}_{\varepsilon}(0)=0$ and the constant $C$ depends only on $k_{0}, T$ and the bounds $\bar{\gamma}=\|\gamma\|_{L^{\infty}(0,1)}$,

$$
\bar{\alpha}=\max \left\{\|\alpha\|_{L^{\infty}\left(Q_{T}\right)},\|\dot{\alpha}\|_{L^{\infty}\left(Q_{T}\right)}\right\}, \quad \beta_{c}=\min \left\{c^{2}, \tilde{\beta}\right\} .
$$

Now using the bounds for $\Psi_{\varepsilon}, \Phi_{\varepsilon}$ and $J_{\varepsilon}$ in $(2.8)$ and $(2.10)$ we derive from (2.23) that

$$
\begin{aligned}
& \left\|\ddot{E}_{\varepsilon}(t)\right\|^{2}+\frac{\beta_{c}}{2}\left\|\dot{E}_{\varepsilon}(t)\right\|_{V}^{2}+2 c \int_{0}^{t}\left|\ddot{E}_{\varepsilon}(\tau, 0)\right|^{2} d \tau \\
\leq & C\left(\|\Phi\|_{H^{2}(0,1)}^{2}+\|\Psi\|_{H^{1}(0,1)}^{2}+\|J\|_{H^{2}\left(0, T ; V^{*}\right)}^{2}\right) \\
& +C \int_{0}^{t}\left(\left\|\dot{E}_{\varepsilon}(\tau)\right\|^{2}+\left\|\ddot{E}_{\varepsilon}(\tau)\right\|^{2}\right) d \tau
\end{aligned}
$$

for sufficiently small $\varepsilon$. Then applying Gronwall's inequality gives immediately the following bound

$$
\left\|\ddot{E}_{\varepsilon}\right\|_{C(0, T ; H)}+\left\|\dot{E}_{\varepsilon}\right\|_{C(0, T ; V)}^{2}+\left\|\ddot{E}_{\varepsilon}(\cdot, 0)\right\|_{L^{2}(0, T)} \leq C
$$

where the constant $C$ is independent of $\varepsilon$. On the other hand, we can similarly obtain the following bounds for the first order derivative of $E_{\varepsilon}$ :

$$
\left\|\dot{E}_{\varepsilon}\right\|_{C(0, T ; H)}+\left\|E_{\varepsilon}\right\|_{C(0, T ; V)}+\left\|\dot{E}_{\varepsilon}(\cdot, 0)\right\|_{L^{2}(0, T)} \leq C .
$$

Using these bounds, we conclude there exists a subsequence which we again denote by $E_{\varepsilon}$ such that as $\varepsilon$ tends to zero,

$$
\begin{aligned}
E_{\varepsilon} & \rightarrow E_{1} & \text { weakly in } & L^{2}(0, T ; V), \\
\ddot{E}_{\varepsilon} & \rightarrow E_{2} & \text { weakly in } & L^{2}(0, T ; H), \\
\dot{E}_{\varepsilon} & \rightarrow E_{3} & \text { weakly in } & L^{2}(0, T ; V), \\
\ddot{E}_{\varepsilon}(\cdot, 0) & \rightarrow E_{0} & \text { weakly in } & L^{2}(0, T) .
\end{aligned}
$$


Using the simple formula

$$
w(t)=w(0)+\int_{0}^{t} \dot{w}(\tau) d \tau
$$

with $w=E_{\varepsilon}$ and $E_{\varepsilon}$, one can readily show that

$$
E_{0}=\ddot{E}_{1}(\cdot, 0), \quad E_{2}=\ddot{E}_{1}, \quad E_{3}=\dot{E}_{1} .
$$

Now letting $\varepsilon$ tend to zero in $(2.12)$, we easily see that $E_{1}$ must be the unique solution $E$ to the problem (2.2). The desired regularity follows from that for $E_{1}$.

Remark 2.1 The consistency conditions and regularity requirement on the initial value functions $\Phi$ and $\Psi$ in Theorem 2.2 are trivially satisfied if there is no initial electric field $E$.

\section{Semi-discrete finite element discretization}

This section will be devoted to the finite element discretization of the variational problem (2.1)-(2.2). For this purpose, we divide the interval $[0,1]$ into $(N+1)$ subintervals of equal size $h=1 /(N+1)$ :

$$
\mathcal{T}^{h}: 0=z_{0}<z_{1}<\cdots<z_{N}<z_{N+1}=1 \text {. }
$$

Let $V_{h}$ be the usual piecewise linear finite element space defined on the partition $\mathcal{T}^{h}$ with all functions vanishing at the boundary node $z=1$, and write $V_{h}$ as

$$
V_{h}=\operatorname{span}\left\{\phi_{1}, \phi_{2}, \cdots, \phi_{N}\right\},
$$

where $\left\{\phi_{i}\right\}$ is the set of the standard piecewise linear nodal basis functions, i.e., $\phi_{i}\left(z_{j}\right)=\delta_{i j}$.

For our later use, we introduce a quasi- $L^{2}$ projection operator $P_{h}: V^{*} \rightarrow$ $V_{h}$ given by

$$
\left(P_{h} w, v_{h}\right)_{h}=\left\langle w, v_{h}\right\rangle \quad \forall w \in V^{*}, v_{h} \in V_{h}
$$

where as already noted, $\langle\cdot, \cdot\rangle$ is the duality pairing between $V$ and $V^{*}$ and $(\cdot, \cdot)_{h}$ is defined by

$$
\left(u_{h}, v_{h}\right)_{h}=\int_{0}^{1} \mathrm{I}_{h}\left(u_{h} v_{h}\right)(x) d x
$$


Here $\mathrm{I}_{h}$ is the nodal value interpolation operator associated with the finite element space $V_{h}$. It is easy to verify that the quasi- $L^{2}$ projection $P_{h} w$ is well-defined for any function $w \in V^{*}$. We will need the following well known properties of the operator $P_{h}$ (see Nochetto-Verdi [11], Xu [14]):

$$
\begin{array}{cl}
\left\|P_{h} w\right\| \leq C\|w\|, & \\
\left\|P_{h} w\right\|_{V} \leq C\|w\|_{V}, & \lim _{h \rightarrow 0}\left\|w-P_{h} w\right\|_{V}=0, \quad \forall w \in H_{R}^{1}(0,1) .
\end{array}
$$

By definition and some simple manipulations we can express $P_{h}$ as

$$
P_{h} w(x)=\sum_{i=1}^{N} q_{i} \phi_{i}(x),
$$

and the coefficients $q_{i}$ are given by

$$
q_{1}=\frac{2}{h}<w, \phi_{1}>, \quad q_{i}=\frac{1}{h}<w, \phi_{i}>\quad \text { for } \quad i \neq 1
$$

We see the quasi- $L^{2}$ projection is very readily computed.

With the above notation, the semi-discrete finite element discretization to the problem (2.1)-(2.2) reads as follows: Find $E_{h}(t) \in V_{h}$ such that

$$
E_{h}(0)=P_{h} \Phi, \quad \dot{E}_{h}(0)=P_{h} \Psi
$$

and for $t \in(0, T)$, the following holds

$$
\begin{aligned}
& \left\langle\tilde{\varepsilon}_{r} \ddot{E}_{h}(t), \phi\right\rangle+\left(\gamma \dot{E}_{h}(t), \phi\right)+\left(\tilde{\beta} E_{h}(t), \phi\right)+\left(G\left(E_{h}\right)(t), \phi\right)+c^{2}\left(E_{h}^{\prime}(t), \phi^{\prime}\right) \\
& +c \dot{E}_{h}(t, 0) \phi(0)=\langle J(t), \phi\rangle+\left(k_{0} E_{h}(t), \phi\right) \quad \forall \phi \in V_{h} .
\end{aligned}
$$

Problem (3.30)-(3.31) is a system of ordinary differential equations and by standard theory we can establish the following theorem.

Theorem 3.1 Under the assumptions of Theorem 2.1, for each fixed $h>0$, the finite element approximation (3.30)-(3.31) has a unique solution $E_{h} \in$ $H^{2}\left(0, T ; V_{h}\right)$. 


\section{Stability estimates and weak convergence}

In this section we shall present some stability estimates for the finite element approximation (3.30)-(3.31) and show the weak convergence of the approximation. First we have

Lemma 4.1 Let $E_{h}(t)$ be the finite element solution to the system (3.30)(3.31). Then under the assumptions of Theorem 2.1, we have

$$
\left\|\dot{E}_{h}\right\|_{C(0, T ; H)}+\left\|E_{h}\right\|_{C(0, T ; V)}+\left\|\dot{E}_{h}(\cdot, 0)\right\|_{H^{1}(0, T)} \leq C .
$$

Proof. The proof is very standard (e.g., see [3]). It can be carried out by taking $\phi=\dot{E}_{h}$ as a test function in equation (3.31), integrating both sides of the resultant equation, using properties (3.26)-(3.27) of the quasi- $L^{2}$ operator $P_{h}$ and invoking Gronwall's inequality.

Using Lemma 4.1 and standard arguments similar in spirit to those in [3], [6] (pp. 100-101), [10] (pp. 272-278) and [13] (pp. 439-442), we can readily prove the following weak convergence.

Theorem 4.1 Under the assumptions of Theorem 2.1, the finite element solution $E_{h}$ to (3.30)-(3.31) converges weakly to a limiting function $E$ in $L^{2}(0, T ; V)$, which is the unique solution to (D.1)-(D.2). Moreover, $\dot{E}_{h}(\cdot, 0)$ converges weakly to $\dot{E}(\cdot, 0)$ in $L^{2}(0, T)$.

Proof. By the boundedness of $E_{h}$ guaranteed in Lemma 4.1, we know immediately that there exists a subsequence, which we again denote by $\left\{E_{h}\right\}$, such that when $h$ tends to zero,

$$
\begin{array}{rlrl}
E_{h} & \rightarrow E & \text { weakly in } & L^{2}(0, T ; V), \\
\dot{E}_{h} \rightarrow E_{1} & \text { weakly in } & L^{2}(0, T ; H), \\
\dot{E}_{h}(\cdot, 0) & \rightarrow E_{0} & \text { weakly in } & L^{2}(0, T) .
\end{array}
$$

Using the simple formula

$$
w(t)=w(0)+\int_{0}^{t} \dot{w}(\tau) d \tau
$$


with $w=E_{h}$ and $\dot{E}_{h}$ and the approximation properties of $P_{h}$, one can readily show that

$$
E_{0}=\dot{E}(\cdot, 0), \quad E_{1}=\dot{E} .
$$

What remains is to prove the limiting function $E$ is a solution to (2.1)-(2.2). For any $\phi \in V$, we take $\phi_{h}=P_{h} \phi \in V_{h}$ as a test function in (3.31) and then multiply both sides of the equation by a function $\xi(t) \in C^{1}[0, T]$ with $\xi(T)=0$ and integrate over $(0, T)$ to obtain

$$
\begin{aligned}
& -\int_{0}^{T}\left(\tilde{\varepsilon}_{r} \dot{E}_{h}(t), \phi_{h}\right) \dot{\xi}(t) d t-\left(\tilde{\varepsilon}_{r} \dot{E}_{h}(0), \phi_{h}\right) \xi(0)+\int_{0}^{T}\left(\gamma \dot{E}_{h}(t), \phi_{h}\right) \xi(t) d t \\
& +\int_{0}^{T}\left(\beta E_{h}(t), \phi_{h}\right) \xi(t) d t+\int_{0}^{T}\left(\int_{0}^{t} \alpha(t-s) E_{h}(s) d s, \phi_{h}\right) \xi(t) d t \\
& +c^{2} \int_{0}^{T}\left(E_{h}^{\prime}(t), \phi_{h}^{\prime}\right) \xi(t) d t+c \int_{0}^{T} \dot{E}_{h}(t, 0) \phi_{h}(0) \xi(t) d t \\
& =\int_{0}^{T}\left\langle J(t), \phi_{h}\right) \xi(t) d t \quad \forall \phi \in V .
\end{aligned}
$$

Now using the weak convergence of $E_{h}$ and the strong convergence of $\phi_{h}=$ $P_{h} \phi$, we obtain by taking $h \rightarrow 0$ in (4.33) that

$$
\begin{aligned}
& -\int_{0}^{T}\left(\tilde{\varepsilon}_{r} \dot{E}(t), \phi\right) \dot{\xi}(t) d t-\left(\tilde{\varepsilon}_{r} \Psi, \phi\right) \xi(0)+\int_{0}^{T}(\gamma \dot{E}(t), \phi) \xi(t) d t \\
& +\int_{0}^{T}(\beta E(t), \phi) \xi(t) d t+\int_{0}^{T}\left(\int_{0}^{t} \alpha(t-s) E(s) d s, \phi\right) \xi(t) d t \\
& +c^{2} \int_{0}^{T}\left(E^{\prime}(t), \phi^{\prime}\right) \xi(t) d t+c \int_{0}^{T} \dot{E}(t, 0) \phi(0) \xi(t) d t \\
& =\int_{0}^{T}\langle J(t), \phi\rangle \xi(t) d t \quad \forall \phi \in V .
\end{aligned}
$$

To see (4.34), let us examine more closely, for example, the convergence of the first and the last terms in the left of (4.33). We have

$$
\begin{aligned}
& -\int_{0}^{T}\left(\tilde{\varepsilon}_{r} \dot{E}_{h}(t), P_{h} \phi\right) \dot{\xi}(t) d t+c \int_{0}^{T} \dot{E}_{h}(t, 0) \xi(t) P_{h} \phi(0) d t \\
= & -\int_{0}^{T}\left(\tilde{\varepsilon}_{r} \dot{E}(t), \phi\right) \dot{\xi}(t) d t+c \int_{0}^{T} \dot{E}(t, 0) \phi(0) \xi(t) d t \\
& -\int_{0}^{T}\left\{\left(\tilde{\varepsilon}_{r} \dot{E}_{h}(t)-\hat{\varepsilon}_{r} \dot{E}(t), \phi\right)+\left(\tilde{\varepsilon}_{r} \dot{E}_{h}(t), P_{h} \phi-\phi\right)\right\} \dot{\xi}(t) d t
\end{aligned}
$$




$$
\begin{aligned}
& +c \int_{0}^{T}\left\{\left(\dot{E}_{h}(t, 0)-\dot{E}(t, 0)\right) \phi(0)+\dot{E}_{h}(t, 0)\left(P_{h} \phi(0)-\phi(0)\right)\right\} \xi(t) d t \\
\rightarrow & -\int_{0}^{T}\left(\tilde{\varepsilon}_{r} \dot{E}(t), \phi\right) \dot{\xi}(t) d t+c \int_{0}^{T} \dot{E}(t, 0) \phi(0) \xi(t) d t .
\end{aligned}
$$

by means of the stability bounds of $E_{h}$ in (4.32), weak convergence of $\dot{E}_{h}$

to $\dot{E}$ and strong convergence of $\phi_{h}=P_{h} \phi$ to $\phi$. Now rewriting the first two terms of (4.34) using integration by parts as

$$
\int_{0}^{T}\left\langle\tilde{\varepsilon}_{r} \ddot{E}(t), \phi\right\rangle \xi(t) d t+\left\{\left(\tilde{\varepsilon}_{r} \dot{E}(0), \phi\right)-\left(\tilde{\varepsilon}_{r} \Psi, \phi\right)\right\} \xi(0),
$$

we see immediately from (4.34) and the arbitrariness of $\xi \in C^{1}[0, T]$ that $\dot{E}(0)=\Psi$ and $E$ satisfies equation (2.2). That is, $E$ is the unique solution of (2.1)-(2.2).

\section{Strong convergence of the finite element solution}

In the preceding section we have proved the weak convergence of the finite element solution $E_{h}$ defined by (3.30)-(3.31) to the solution $E$ of (2.1)-(2.2) under very mild regularity properties for $E$ that are guaranteed by the existence results in Theorem 2.1. However we note in Theorem 2.2 that we can gain additional regularity of solutions in the time variable by using the special structure of the input source function. This structure permits us to assume that it is reasonablly smooth in the time variable although it is a distribution in the space variable. Using this higher regularity in the time variable we are able to prove strong convergence of the finite element solution $E_{h}$. For this purpose, it suffices to show the strong convergence of $\xi_{h} \equiv P_{h} E-E_{h}$ by using the relation

$$
E(t, z)-E_{h}(t, z)=\left\{E(t, z)-P_{h} E(t, z)\right\}+\left\{P_{h} E(t, z)-E_{h}(t, z)\right\}
$$

and the approximation properties of the quasi- $L^{2}$ projection operator $P_{h}$ in (3.26)-(3.27).

Our next aim is to show the strong convergence of $\xi_{h}$ under the assumptions of Theorem 2.2. For this purpose we subtract equation (3.31) from 
equation (2.2) with a $\phi \in V_{h}$ as a test function to obtain

$$
\begin{aligned}
& \left(\tilde{\varepsilon}_{r}\left(\ddot{E}-\ddot{E}_{h}\right), \phi\right)+\left(\gamma\left(\dot{E}-\dot{E}_{h}, \phi\right)+\left(\tilde{\beta}\left(E-E_{h}, \phi\right)\right.\right. \\
& +\left(\int_{0}^{t} \alpha(t-s)\left(E(s)-E_{h}(s)\right) d s, \phi\right)+c^{2}\left(\left(E-E_{h}\right)^{\prime}, \phi^{\prime}\right) \\
& +c\left(\dot{E}-\dot{E}_{h}\right)(t, 0) \phi(0)=k_{0}\left(E-E_{h}, \phi\right) \quad \forall \phi \in V_{h},
\end{aligned}
$$

which can be written by some simple arrangements as

$$
\begin{aligned}
& \left(\tilde{\varepsilon}_{r} \ddot{\xi}_{h}(t), \phi\right)+\left(\tilde{\beta} \xi_{h}(t), \phi\right)+c^{2}\left(\xi_{h}^{\prime}(t), \phi^{\prime}\right)+c \dot{\xi}_{h}(t, 0) \phi(0) \\
= & \left(\gamma\left(\dot{E}_{h}-\dot{E}\right)(t), \phi\right)+\left(\int_{0}^{t} \alpha(t-s)\left(E_{h}-E\right)(s) d s, \phi\right) \\
& +\left(k_{0}\left(E_{h}-E\right)(t), \phi\right)+\left(\tilde{\varepsilon}_{r} P_{h} \ddot{E}(t)-\tilde{\varepsilon}_{r} \ddot{E}(t), \phi\right)+c\left(P_{h} \dot{E}-\dot{E}\right)(t, 0) \phi(0) \\
& +\left\{\left(\tilde{\beta} P_{h} E(t)-\tilde{\beta} E(t), \phi\right)+c^{2}\left(\left(P_{h} E-E\right)^{\prime}(t), \phi^{\prime}\right)\right\} \\
\equiv: & \sum_{i=1}^{6}(\mathrm{II})_{i} .
\end{aligned}
$$

Taking $\phi=\dot{\xi}_{h}$ in (5.37), we find that the left side of (5.37) equals

$$
\frac{1}{2} \frac{d}{d t}\left\{\left(\tilde{\varepsilon}_{r} \dot{\xi}_{h}(t), \dot{\xi}_{h}(t)\right)+\left(\tilde{\beta} \xi_{h}(t), \xi_{h}(t)\right)+c^{2}\left\|\xi_{h}^{\prime}(t)\right\|^{2}\right\}+c\left|\dot{\xi}_{h}(t, 0)\right|^{2}
$$

Thus integrating $(5.37)$ over $(0, t)$ we obtain

$$
\left\|\dot{\xi}_{h}(t)\right\|^{2}+\beta_{c}\left\|\xi_{h}(t)\right\|_{V}^{2}+2 c \int_{0}^{t}\left|\dot{\xi}_{h}(\tau, 0)\right|^{2} d \tau \leq 2 \sum_{i=1}^{6} \int_{0}^{t}(\mathrm{II})_{i} d \tau
$$

where we have used the facts that $\tilde{\varepsilon}_{r} \geq 1, \xi_{h}(0)=\dot{\xi}_{h}(0)=0$. We next derive bounds for each of the terms $\int_{0}^{t}(\mathrm{II})_{i} d \tau$ in (5.38).

First, by the definition of $\xi_{h}$ and the Cauchy-Schwarz inequality along with bounds $\bar{\gamma}, \bar{\alpha}$ and $\varepsilon_{r}$ for $\gamma, \alpha$ and $\tilde{\varepsilon}_{r}$ we obtain

$$
\begin{aligned}
\int_{0}^{t}(\mathrm{II})_{1} d \tau & =-\int_{0}^{t}\left(\gamma \dot{\xi}_{h}(\tau), \dot{\xi}_{h}(\tau)\right) d \tau+\int_{0}^{t}\left(\gamma\left(P_{h} \dot{E}-\dot{E}\right)(\tau), \dot{\xi}_{h}(\tau)\right) d \tau \\
& \leq \frac{3 \bar{\gamma}}{2} \int_{0}^{t}\left\|\dot{\xi}_{h}(\tau)\right\|^{2} d \tau+\frac{\bar{\gamma}}{2} \int_{0}^{t}\left\|\left(\dot{E}-P_{h} \dot{E}\right)(\tau)\right\|^{2} d \tau
\end{aligned}
$$




$$
\begin{aligned}
\int_{0}^{t}(\mathrm{II})_{2} d \tau & \leq \int_{0}^{t} \int_{0}^{\tau} \bar{\alpha}\left\|E_{h}(s)-E(s)\right\|\left\|\dot{\xi}_{h}(\tau)\right\| d s d \tau \\
& \leq \bar{\alpha} \int_{0}^{t} \int_{0}^{\tau}\left\|\dot{\xi}_{h}(\tau)\right\|\left(\left\|\xi_{h}(s)\right\|+\left\|\left(E-P_{h} E\right)(s)\right\|\right) d s d \tau \\
& \leq t \bar{\alpha} \int_{0}^{t}\left(\left\|\dot{\xi}_{h}(\tau)\right\|^{2}+\left\|\xi_{h}(\tau)\right\|^{2}\right) d \tau+\frac{t \bar{\alpha}}{2} \int_{0}^{t}\left\|\left(E-P_{h} E\right)(\tau)\right\|^{2} d \tau, \\
\int_{0}^{t}(\mathrm{II})_{3} d \tau & \leq k_{0} \int_{0}^{t}\left(\left\|\dot{\xi}_{h}(\tau)\right\|^{2}+\left\|\xi_{h}(\tau)\right\|^{2}\right) d \tau+\frac{k_{0}}{2} \int_{0}^{t}\left\|\left(E-P_{h} E\right)(\tau)\right\|^{2} d \tau, \\
\int_{0}^{t}(\mathrm{II})_{4} d \tau & \leq \frac{1}{2} \int_{0}^{t}\left\|\dot{\xi}_{h}(\tau)\right\|^{2} d \tau+\frac{\varepsilon_{r}^{2}}{2} \int_{0}^{t}\left\|P_{h} \ddot{E}(\tau)-\ddot{E}(\tau)\right\|^{2} d \tau, \\
\int_{0}^{t}(\mathrm{II})_{5} d \tau & \leq \frac{c}{2} \int_{0}^{t}\left|\dot{\xi}_{h}(\tau, 0)\right|^{2} d \tau+\frac{c}{2} \int_{0}^{t}\left|\left(P_{h} \dot{E}-\dot{E}\right)(\tau, 0)\right|^{2} \\
& \leq \frac{c}{2} \int_{0}^{t}\left|\dot{\xi}_{h}(\tau, 0)\right|^{2} d \tau+C \int_{0}^{t}\left\|\left(P_{h} \dot{E}-\dot{E}\right)(\tau)\right\|_{V}^{2} d \tau
\end{aligned}
$$

where we have used the continuous embedding of $H^{1}(0,1)$ into $C[0,1]$ in the last inequality. Finally for the term $\int_{0}^{t}(\mathrm{II})_{6} d \tau$, we have by integration by parts

$$
\begin{aligned}
\int_{0}^{t}(\mathrm{II})_{6} d \tau= & \int_{0}^{t}\left(\tilde{\beta} P_{h} E(\tau)-\tilde{\beta} E(\tau), \dot{\xi}_{h}(\tau)\right) d \tau-c^{2} \int_{0}^{t}\left(\left(P_{h} \dot{E}-\dot{E}\right)^{\prime}(\tau), \xi_{h}^{\prime}(\tau)\right) d \tau \\
& +c^{2}\left(\left(P_{h} E-E\right)^{\prime}(t), \xi_{h}^{\prime}(t)\right)-c^{2}\left(\left(P_{h} E-E\right)^{\prime}(0), \xi_{h}^{\prime}(0)\right) \\
\leq & \frac{\bar{\beta}_{c}}{2} \int_{0}^{t}\left(\left\|P_{h} E(\tau)-E(\tau)\right\|^{2}+\left\|\left(P_{h} \dot{E}(\tau)-\dot{E}\right)^{\prime}(\tau)\right\|^{2}\right) d \tau \\
& +\frac{\bar{\beta}_{c}}{2} \int_{0}^{t}\left(\|\dot{\xi}(\tau)\|^{2}+\left\|\xi_{h}^{\prime}(\tau)\right\|^{2}\right) d \tau+\frac{\beta_{c}}{4}\left\|\xi_{h}^{\prime}(t)\right\|^{2} \\
& +\frac{c^{4}}{\beta_{c}}\left\|\left(P_{h} E-E\right)^{\prime}(t)\right\|^{2}
\end{aligned}
$$

with $\bar{\beta}_{c}=\max \left\{c^{2},\|\tilde{\beta}\|_{L^{\infty}(0,1)}\right\}$. Substituting all the bounds for $\int_{0}^{t}(\mathrm{II})_{i} d \tau$ into equation (5.38), we have

$$
\begin{aligned}
& \left\|\dot{\xi}_{h}(t)\right\|^{2}+\frac{\beta_{c}}{2}\left\|\xi_{h}(t)\right\|_{V}^{2}+c \int_{0}^{t}\left|\dot{\xi}_{h}(\tau, 0)\right|^{2} d \tau \\
& \leq \delta_{h}+C \int_{0}^{t}\left(\left\|\dot{\xi}_{h}(\tau)\right\|^{2}+\left\|\xi_{h}(\tau)\right\|^{2}+\left\|\xi_{h}^{\prime}(\tau)\right\|^{2}\right) d \tau
\end{aligned}
$$

with $\delta_{h}$ given by

$$
\delta_{h}=C_{0}\left\{\int _ { 0 } ^ { T } \left(\left\|\left(\dot{E}-P_{h} \dot{E}\right)(t)\right\|^{2}+\left\|\left(E-P_{h} E\right)(t)\right\|^{2}+\left\|P_{h} \ddot{E}(t)-\ddot{E}(t)\right\|^{2}\right.\right.
$$




$$
\left.\left.+\left\|\left(P_{h} \dot{E}-\dot{E}\right)(t)\right\|_{V}^{2}\right) d t+\left\|P_{h} E-E\right\|_{C(0, T ; V)}^{2}\right\} .
$$

We know that $\delta_{h}$ tends to zero by means of the approximation properties (3.26)-(3.27). Thus applying Gronwall's inequality to (5.41) we finally obtain

$$
\left\|\dot{\xi}_{h}\right\|_{C(0, T ; H)}+\left\|\xi_{h}\right\|_{C(0, T ; V)}+\left\|\dot{\xi}_{h}(\cdot, 0)\right\|_{L^{2}(0, T)} \rightarrow 0 \quad \text { as } \quad h \rightarrow 0 .
$$

This along with (5.35) and the approximation properties (3.26)-(3.27) again yields

Theorem 5.1 Let $E$ and $E_{h}$ be the solutions to (2.1)-(2.2) and (3.30)-(3.31) respectively. Then under the assumptions of Theorem $2.2, E_{h}$ converges to $E$ strongly in spaces $H^{1}(0, T ; H)$ and $C(0, T ; V)$, and $E_{h}(\cdot, 0)$ converges strongly to $E(\cdot, 0)$ in $H^{1}(0, T)$.

Acknowledgements. The authors are grateful to Dr. Richard A1banese, Air Force Research Labs, Brooks AFB, for numerous fruitful communications and suggestions regarding the model discussed in this paper.

\section{References}

[1] R. Albanese and J. Penn and R. Medina, Short-rise-time microwave pulse propagation through dispersive biological media, J. Optical Soc. Amer. A, 6 (1989), pp. 1441-1446.

[2] C.A. Balanis, Advanced Engineering Electromagnetics, J. Wiley, New York, 1989.

[3] H.T. Banks and M.W. Buksas, Electromagnetic interrogation of dielectric materials, Technical Report CRSC-TR98-30, Center for Research in Scientific Computation, North Carolina State University, August, 1998

[4] H.T. Banks and M.W. Buksas and Y. Wang, A time domain formulation for identification in electromagnetic dispersion, Technical Report CRSC-TR96-30, Center for Research in Scientific Computation, North Carolina State University, Oct., 1996; J. Math. Systems, Est. and Control, 8 (1998), pp. 257-260. 
[5] H.T. Banks and T. Lin, Determining the structure of a biological medium using accoustic probes, Technical Report CRSC-TR98-21, Center for Research in Scientific Computation, North Carolina State University, May, 1998; Submitted to J. Inverse and Ill-posed Problems.

[6] H.T. Banks and R.C. Smith and Y. Wang, Smart Material Structures: Modelling, Estimation and Control, Wiley/Masson, Chichester/Paris, 1996.

[7] R.S. Beezley and R.J. Krueger, An electromagnetic inverse problem for dispersive media, J. Math. Phys., 26 (1985), pp. 317-325.

[8] V.V. Daniel, Dielectric Relaxation, Academic Press, New York, 1967.

[9] J.D. Jackson, Classical Electrodynamics, Second edition, John Wiley and Sons, New York, 1975.

[10] J.L. Lions, Optimal Control of Systems Governed by Partial Differential Equations, Springer-Verlag, New York, 1971.

[11] R.H. Nochetto and C. Verdi, Approximation of degerate parabolic problems using numerical integration, SIAM J. Numer. Anal., 25 (1988), pp. 784-814.

[12] T.M. Roberts and M. Hobart, Energy velocity, damping and elementary inversion, J. Opt. Soc. Amer. A, 9 (1992), pp. 1091-1101.

[13] J. Wloka, Partial Differential Equations, Cambridge University Press, Cambridge 1987.

[14] J. Xu, Theory of Multilevel Methods, Ph.D. dissertation, Department of Mathematics, Cornell University (1989). 